\title{
Probability in the Many-Worlds Interpretation of Quantum Mechanics
}

\author{
Lev Vaidman
}

April 7, 2011

\begin{abstract}
It is argued that, although in the Many-Worlds Interpretation of quantum mechanics there is no "probability" for an outcome of a quantum experiment in the usual sense, we can understand why we have an illusion of probability. The explanation involves: a). A "sleeping pill" gedanken experiment which makes correspondence between an illegitimate question: "What is the probability of an outcome of a quantum measurement?" with a legitimate question: "What is the probability that "I" am in the world corresponding to that outcome?"; b). A gedanken experiment which splits the world into several worlds which are identical according to some symmetry condition; and c). Relativistic causality, which together with (b) explain the Born rule of standard quantum mechanics. The Quantum Sleeping Beauty controversy and "caring measure" replacing probability measure are discussed.
\end{abstract}

\section{Introduction}

Itamar and I shared a strong passion for understanding quantum mechanics. We did not always view it in the same way but I think we understood each other well. In fact I am greatly indebted to Itamar. Being a physicist working on foundations of quantum mechanics I always thought that philosophical arguments are crucial for understanding quantum mechanics. However, my first philosophical work [1] was rejected over and over by philosophical journals and philosophers. While Hillary Putnam, Abner Shimony, 
Michael Redhead and others did not see the point I was making, it was Itamar who first appreciated my contribution and opened for me the way to the philosophy of science [2].

There are three conceptually different scenarios of what happens in the process of quantum measurement. The first option is that there is a genuinely random (chance) event which makes one outcome happen without any possibility to know which one prior to the measurement. This is the case of collapse: the von Neumann type II evolution of the quantum wave, or the stochastic event in a theory of dynamical collapse [3, 4]. The second option is that the quantum wave description of the the system is deterministic, there is no collapse, but it is incomplete. There are hidden variables specifying the outcome prior to the measurement, which, however, we cannot know in principle. The most successful proposal of this kind is causal interpretation [5]. The third option is that the evolution is deterministic, there is no collapse of the quantum wave and the quantum wave is the complete description of the system. Then, all outcomes take place and this is the many-worlds interpretation (MWI) [6].

The concept of probability is directly applicable in the first scenario. There is genuine chance and genuine uncertainty. If, say, $A$ is a possible outcome, then we can talk about the probability that $A$ will happen. Indeed, $A$ might or might not happen. At the end of the process we will definitely know if $A$ took place.

In the second scenario there is no random chance. Prior to experiment Nature knows the outcome, it is encoded in some (hidden) variable. There are no several options, only one. However, since the theory postulates that "hidden variables" cannot be known to the experimentalist, he has an ignorance-type probability: he does not know the value of the hidden variable which specifies the outcome of the experiment. His concept of probability is: the probability that $A$ will happen is the probability that the hidden variables now are such that $A$ will take place.

The situation is the most difficult in the third scenario. There is no randomness, there is no chance: $A$ happens with certainty, but other non compatible outcomes happen with certainty too, so a standard concept of probability addressing the dilemma $A$ or not $A$ is not applicable here. We have no uncertainty, everything is known. We have a complete description prior to the measurement and the process of measurement is some known deterministic evolution; so we know the complete description now and forever. These leads us to the conclusion that we do not have probability here in the 
usual sense. But this can be expected since the picture of multiple worlds is rather unusual. I will argue that we have here an illusion of probability, an illusion behaving very much like the usual probability.

\section{The "Tale of a single-world Universe"}

Before starting the analysis of probability in the MWI I have to clarify exactly what is the MWI since it has numerous, sometimes contradictory, presentations in the literature. The MWI, as I understand it [7], is the claim that All is the Universal Wave Function evolving according to the laws of standard quantum mechanics without collapse, together with the explanation of the correspondence between the Wave Function and our experience.

In order to explain our experience I find it useful to introduce the "Tale of a single-world Universe". Let us assume that we are the only civilization and that we live under a very strong dictatorship which has laws against quantum measurements. It is forbidden to perform quantum experiments in which there is a nonzero probability for more than one outcome. Manufacture of Geiger counters is banned, quantum random number generators [8] are forbidden, and a special police prevents world splitting devices of the kind that can be found in Tel-Aviv university [9]. There are even laws that under the threat of death enforce disposal of neon light bulbs after six months of operation, to avoid operating an old bulb, which, when flicking, splits our world.

In this tale Nature does not arrange quantum experiments accidentally: no macroscopically different superpositions of a macroscopic object ever develop. In such a Universe there is no difference between the MWI and the textbook interpretation: in both, the wave function evolves according to the Schrödinger equation since collapse takes place in the measurement-type situations, but in our tale these situations never take place. The wave functions of all macroscopic objects remain well localized all the time.

The connection of the Wave Function to our experience in such a Universe is through a three dimensional picture which is generated by the Wave Function. Indeed, the three dimensional map of the density of wave functions of all particles will form a familiar picture of macroscopic objects around us as well as our bodies moving in time in a classical manner.

I am aware that there are claims that the Wave Function cannot describe the reality because it is defined in configuration space [10]. In classical me- 
chanics a similar complaint is easily rejected because we can consider each particle separately in three dimensional space, instead of one point in the configuration space of all $N$ particles. In quantum mechanics it is more difficult, since we cannot neglect entanglement. Although in our tale macroscopic bodies are never entangled, electrons are surely entangled with nuclei in atoms and atoms entangled in molecules. Still, the picture is in three dimensions. Even if an electron in my finger is entangled with nuclei and other atoms in the molecule of my skin, its density in three dimensions is well localized. This picture in three dimensional space is what corresponds to our observations. What makes the representation of the Wave Function in space special, relative to some abstract Hilbert space representation, is that the interactions in Nature are local. Since our observations are also kind of interactions, they are local too.

\section{Illusion of probability}

Once we understand the link between the Wave Function and experience in a single-world Universe, we can proceed to analyze a Universe in which quantum measurements are not forbidden. The quantum measurements will lead to a superposition of branches of the Wave Function, each one of them corresponding to what we experience as a "world". Until the next measurement, the link with our observations works in each branch as in a single-world Universe. The locality and strength of interactions in Nature ensure that parallel branches do not interfere (decoherence). Given the information we have at present, we can follow our branch to the past before the last quantum measurement, but we can follow it to the future only until the next measurement. In our branch we remember past events of performing quantum measurements and obtaining particular results. It seems to us that the outcomes came out randomly, although we know that there were no random evolution in Nature. The branch was split deterministically to two or more branches. We now experience only one of them and it seems to us that there was a random outcome of the quantum measurement.

If we imagine a hypothetical theory in which the wave function collapses every time a macroscopic object evolves into a superposition of macroscopically different states such that all macroscopic objects (whatever "macroscopic" means) remain well localized, then the memory and experience of the observers in the single world described by such a theory will be identical 
to the experience of observers in one branch of the many-world universe. In the Universe with collapse there is a genuine probability concept of random chancy events. In the MWI universe there is deterministic evolution, and no objective "chancy" probability. But the experiences in a particular branch (we can follow the branch in the MWI backwards in time) are identical to the experiences of genuine probability of the observers living in the physical universe with collapses of the quantum wave. This explains the illusion of probability in the MWI.

So, one approach to introducing "probability" to the MWI is to point out that the observer in a branch of the Universe in the framework of the MWI and the observer in the single-world Universe with collapse postulate are described by the same mathematical object and thus have the same experience. Since in the theory with collapse, the probability concept is clear, we can associate the same concept in the branch of the MWI with the observer who is planning to perform a quantum experiment.

Although I do not think that the probability can be derived in the framework of the MWI as Deutsch advocates [11], I do think that one can argue more why the illusion of probability in the MWI works so well. To illustrate this let us consider two gedanken experiments. The experiments will include steps which seem technologically unimaginable, yet they do not require changes in any physical law.

\section{Gedanken Experiment I: complete symme- try}

Three identical space stations $A, B$ and $C$ were built and put on the same orbit around Earth in a symmetrical way, see Fig.1. Bob wants to travel to space and he arranges an automatic device which will send him to one of these stations after he goes to sleep. The device consists of a spin-1 particle in the state

$$
\frac{1}{\sqrt{3}}(|1\rangle+|0\rangle+|-1\rangle)
$$

measuring device of the spin component and the spaceship which will move him, while he is asleep, to one of the stations according to the the outcome of the spin measurement. Bob, who accepts the MWI of quantum mechanics is certain that, at a later time, there will be three Bobs. The quantum state 

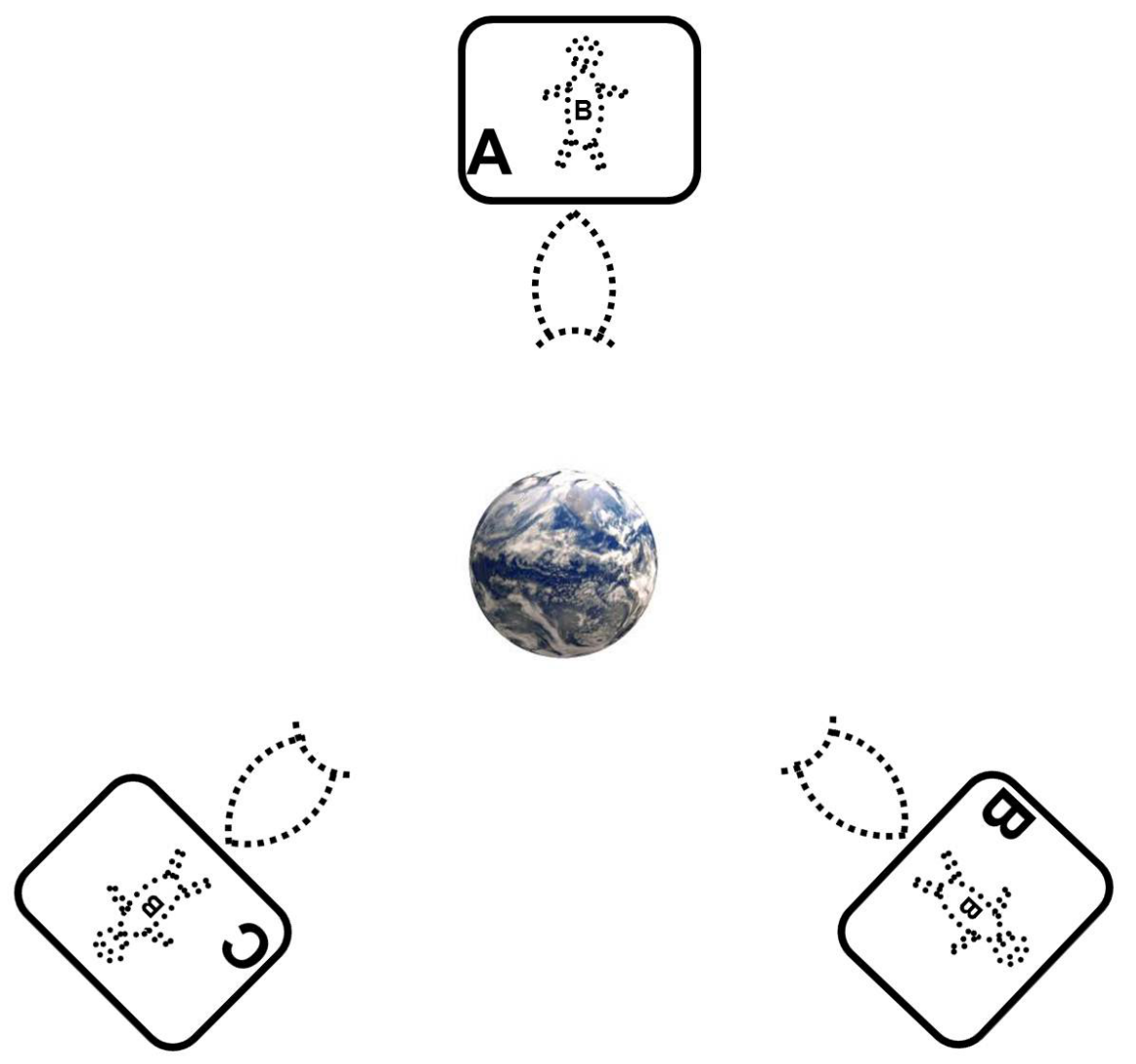

Figure 1: Bob's descendants in symmetric state in three space stations.

will be

$$
\frac{1}{\sqrt{3}}(|A\rangle|1\rangle+|B\rangle|0\rangle+|C\rangle|-1\rangle)
$$

where $|A\rangle$ signifies the quantum state of Bob in $A$ as well as the state of spaceship which brought him to $A$ and everything else which interacted with Bob and his spaceship and became correlated to his wave function in $A$, and similarly for $|B\rangle$ and $|C\rangle$. Bob in $A$ will know that there will be yet other two Bobs at that time, one in $B$ and another in $C$.

While this is a very expensive experiment, it is by no means technologically unthinkable. What makes the experiment more difficult is the requirement of complete identity (apart from their symmetric location on the 
orbit) of the states $|A\rangle,|B\rangle$ and $|C\rangle$. Given this symmetry, each waking Bob will have a genuine concept of probability of being in $A$ equal to one third. They will have the probability concept in spite of the fact that they will know everything about the world, or at least everything about the spaceship, satellites and their bodies and that the complete description of these systems has no random elements. The only thing they will be ignorant about is their identity.

These three Bobs will be in a privileged situation, as only they have a meaningful concept of probability. Due to symmetry between the three Bobs, the probability of being Bob $A$ is one third. Insofar as everyone else is concerned, the probability for a particular Bob to be in $A$ is either 1 or 0 , since the only possibility to identify a particular Bob is according to his location.

Albert [12] was arguing that the probability I constructed here appears too late. He claims that we need to assign probability before performing the experiment, while descendants of the experimentalist obtain their ignorance probability only after the experiment. Indeed, the probability concept of Bob's descendants is not the probability concept for the outcome of the experiment for Bob before the experiment. There is no meaning for Bob, before the experiment, to the question: "What is the probability that Bob will reach space station $A$, since he will reach all stations.

In my opinion, the criticism of Albert falls short because I do not claim that there is a genuine probability in the MWI. There is only an illusion, and all what I am trying to say is that this illusion behaves exactly as if there was a real concept of probability. If we adopt an approach for probability as the value of an "intelligent bet" [13], then Bob makes bets understanding that the consequences of the bet will be relevant for his descendants (Bobs after the experiment). They will get the reward of the bet (and they will have initially less money if Bob spent money on the bet). They will have an ignorance concept of probability, so they will be pleased to find out that the bet was placed. The Bob before the experiment cared about Bobs after the experiment, due to symmetry of the situation, in an equal way. This, together with the fact that all Bobs like to bet, provide the rational for his betting. 


\section{Gedanken Experiment II: Derivation of the Born Rule}

The sleeping pill trick [1] provides the way to talk about probability in the MWI, which, in my view is the main difficulty to be resolved. In recent years, however, even more attention was given to the issue of the Born rule in the framework of the MWI, i.e. not just to justifying the probability concept when all outcomes of the experiment are realized, but also assigning the correct values of probability for different outcomes. In the above idealized symmetric setup we do get the correct probability, one third, but we need to work much more for a general case.

Let us note that even in Experiment I there is no complete symmetry: I gave names to Bob's (identical) stations, one of them is " $A$ " and others are not. In a completely symmetrical situation there cannot be different names. So, the symmetry is not complete. We only assume that all relevant aspects of the three stations are completely identical, but we accept a possibility, and in fact a necessity, that there are other properties of the stations, like pictures on their surface which are different. In a scientific theory we have an idea as to what is relevant and what is not. A hypothesis that a different text drawn on otherwise identical space stations will change the outcome of the experiment described above does not seem to be scientific. So, we have to make our setup symmetric only in relevant details.

Let us modify the above setup trying to keep relevant aspects symmetric, in a way which will lead us to the Born rule. We still have our three identical space stations, but now, the observer, Bob, is moved only to space station $A$. We also send Charlie and John to stations $B$ and $C$ and perform similar operations there to keep at least partially the symmetry between the stations, see Fig.2. While Bob is asleep (as well as Charlie and John), a device of the type described above causes a particle to be in a superposition in all three stations. Then, in all three stations automatic devices perform measurements of the presence of the particle there. According to the outcome of such measurement in station $A$, Bob is moved to a room "yes" if the particle is found in $A$ and to a room "no" if the particle is not found there. Similar operations are performed in stations $B$ and $C$. Now, upon awakening, each Bob will have a genuine ignorance probability concept regarding the question: "In which room am I?" Each Bob will have a reason to declare probability one third for being in the room "yes", because there are three worlds and 

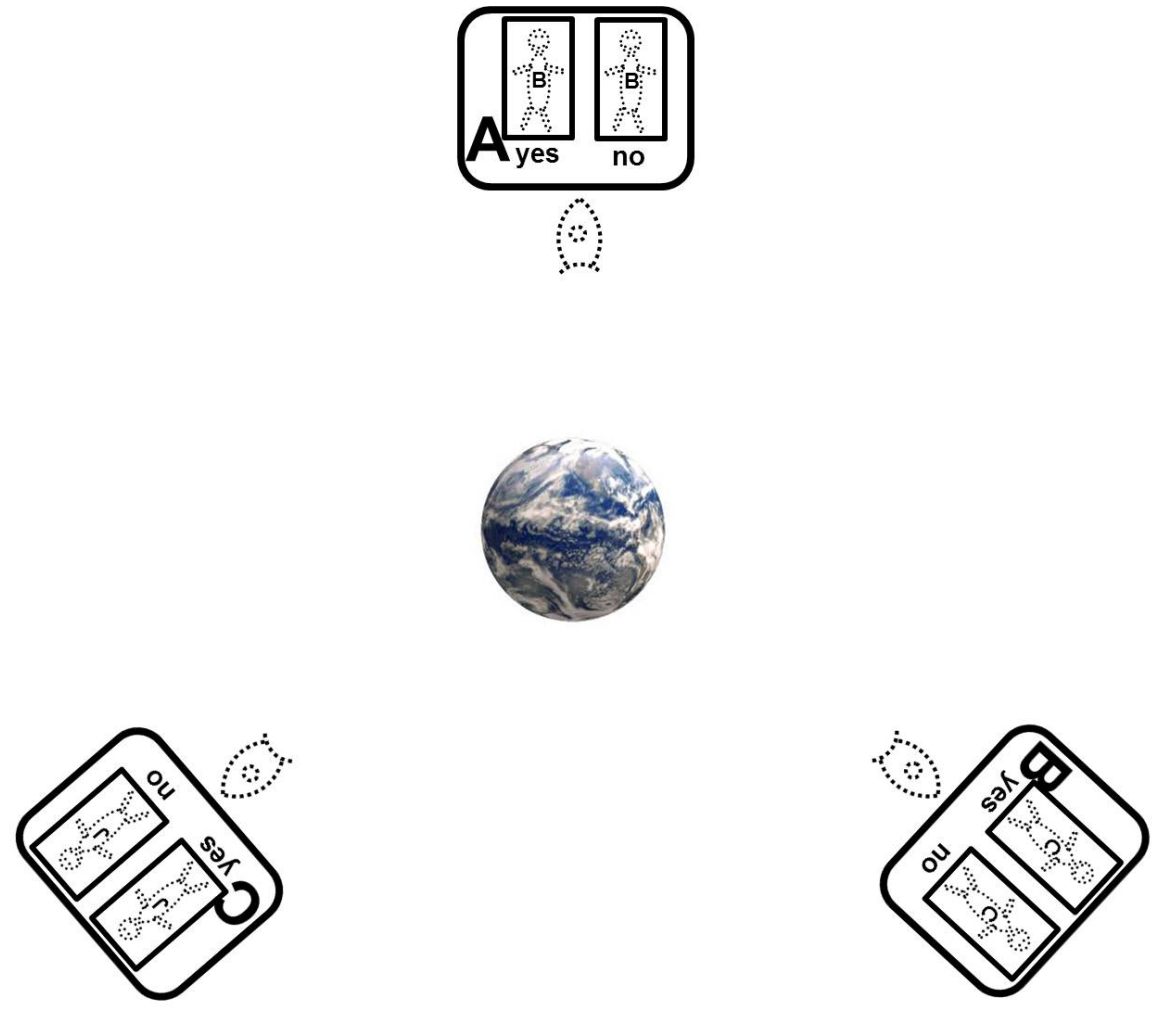

Figure 2: Bob's descendant in room "yes", while Charlie's and John's descendants are in rooms "no" in superposition with two other similar options.

only in one of them this Bob is in the room "yes".

The statement that "there are three worlds" needs clarification. In one world Bob cannot view himself in a superposition, so, it was clear in Experiment I that there are three worlds: in the first Bob is in $A$, in the second he is in $B$, and in the third he is in $C$. In Experiment II, in the first world, $A$, Bob is in room "yes"; in the the worlds $B$ and $C$ he is in the room "no". If we follow Everett's original "Relative state" formulation of quantum mechanics' [6], we might say that for Bob there are only two worlds: in one of them he is in the "yes" room and in another he is in the "no" room. In my approach [7] macroscopic objects and especially people cannot be in a superposition of macroscopically different states in one world. So, the measurements of Charlie and John in stations $B$ and $C$ ensure that there are three worlds: 
$A, B$, and $C$, with Bob, Charlie, and John, in their "yes" rooms correspondingly, while the others are in "no" rooms. The symmetry argument here is suggestive but not rigorous. We need three worlds which are symmetric in all relevant aspects. It is not obvious that the fact that in two of the worlds ( $B$ and $C$ ) there is one and the same Bob, while in the world $A$ there is another Bob, is not relevant for our analysis.

Accepting the suggestive symmetry argument that gives Bob probability one third to find himself in room "yes", provides the Born rule for this case: $p_{\text {yes }}=\frac{1}{3}=\left\langle\mathbf{P}_{A}\right\rangle$. Now we can add to the MWI the locality and causality postulates.

The MWI yields: There is nothing but the wave function.

Locality provides: Outcomes of local experiments depend only on local values of the wave function.

Causality of relativistic quantum theory yields: Any action in a space-like separated region cannot influence an outcome of local experiment.

From this it follows that Bob should assign probability $p_{y e s}=\frac{1}{3}$ for all states which can be obtained from (2) through actions at regions which are space-like separated from the measurement in $A$. For example, if Charlie and John in stations $B$ and $C$ do not perform measurements of the presence of the particle, the symmetry is broken: there will be two worlds instead of three, but the probability to find the particle in $A$ remains one third. This gedanken experiment shows that the probability of finding the particle in $A$ for a quantum state which allows "symmetrization", i.e., there exists symmetric state with $N$ parts with the same density matrix at location $A$, is $\frac{1}{N}$.

A more general question is the probability of an outcome of any quantum measurement in a particular location, not just the measurement of projection operator of a particle on this location. A celebrated example is a Stern Gerlach measurement of a spin component. To cover this case we can consider unitary evolution which creates a spin state via absorbtion of a photon. Then, the spin component measurement is equivalent to a photon projection measurement [14]. The concept of "symmetrization" becomes: existence of a symmetric situation with identical systems in symmetrically located $N$ locations with the same density matrix at location $A$.

Generalizing the argument for an arbitrary state a la Deutsch [11] or by using Gleason theorem [15] we can derive the Born rule. However, I do not see how to make this derivation rigorous. If we could make a similar argument for Experiment I, in which symmetry is robust, this could provide a rigorous 
derivation of the probability in the MWI. However, the locality argument cannot be applied there. Bob is not localized in this experiment. When he is asked what is the probability that he is in $A$, the situation in $B$ and $C$ matters. For example, if Bob knows that nobody will ask this question in $B$ and $C$, he should give the answer 1 instead of $\frac{1}{3}$. (Note that when Charlie and John refrain from making measurements in $B$ and $C$ it changes nothing in Experiment II.)

So, although the Born rule fits the MWI very well, I do not see how to derive it without some (plausible) assumptions of what is relevant for the probability of an outcome of a quantum measurement. But then, similar, if not simpler, symmetry arguments yield the Born rule also in the framework of collapse interpretations, so I do not think that the MWI has an advantage relative to this question. I adopt the MWI because it removes randomness and nonlocality from physics.

\section{Quantum Sleeping Beauty}

It is harder to approach the probability issue in the framework of the MWI than in other interpretations of quantum mechanics and I found only one situation in which the MWI helps to analyze a probabilistic question. This is the story of "Sleeping Beauty" [16].

Some researchers put Beauty to sleep. During the two days of her sleep they will briefly wake her up either once or twice, depending on the toss of a fair coin (Heads: once; Tails: twice). After each waking, they will put Beauty back to sleep with a drug that makes her forget that waking. Every wakening the Beauty is asked: What is your credence for the outcome Heads?

This problem raised a great controversy: is the the answer one third or one half? Although I believe that one can argue convincingly that the answer is one third without help of quantum mechanics [17], the MWI provides an even more convincing argument [18]. I implement the fair coin toss via quantum experiment with probability half, which is an ultimate fair coin. Then one can unambiguously describe the situation as the unitary evolving quantum wave of Beauty and quantum measuring device. This makes the problem easier to analyze than in the case of a "chancy" fair coin. For simplicity I will add to the story that before wakening, the Beauty is moved to the room "Heads" or "Tails" according to the result of the quantum measurement. The rooms are identical inside, so, when she is asked the question she is 
in one of three different locations in space and time, but she will have the same memory state and identical environment. For the case of the quantum measurement, the question: "Is it Heads or Tails?" is senseless, since both options are realized. The actual question is: "Is it Heads or Tails in the world the Beauty is asked the question?" (Compare with Groisman's [19] approach to resolve the Sleeping Beauty controversy for the classical coin.) Beauty knows that in the Universe there are three events in which she is asked this question. The measures of existence of worlds in all these events are equal. Since only one of these events corresponds to Heads, she assigns probability one third for Heads.

The Quantum Sleeping Beauty also generated a considerable controversy. To my surprise the answer one third is not in the consensus. Peter Lewis [20] claimed that quantum coin tossing leads to the Beauty's answer of one half. He insisted, especially, that it has to be one half in the framework of the MWI [21]. Very recently Bradley [22] also claimed (but did not show) that the MWI approach leads to the answer one half, adding that in his view this is good news for the MWI. Papineau and Dura-Vila [23] criticized Lewis, but argued that accepting my approach to probability in the MWI strengthens Lewis's claim for one half.

Most of Lewis's arguments rest on assigning pre-branching uncertainty in the MWI advocated by Saunders [24] and Wallace [25] which I strongly deny. Lewis briefly mentions that one half is obtained in my approach too, arguing that there is an analogy with a process with two consecutive coin tosses. (I just learned that Peterson [26] argued against this analogy.) I could not see such an analogy: the only second coin toss in the Sleeping Beauty story I can imagine is her guess about which wakening, out of three, is now. I cannot understand the rational for Lewis's coin toss between the two Tail wakening. The answer one half seems to follow from an error similar to the Bertrand Box paradox [27].

\section{Caring measure instead of probability}

In the MWI there is no genuine probability. Instead of probability measure, I introduced "measure of existence" of a world. Measure of existence, apart from its relation to probability, describes the ability of a particular world to interfere with other worlds [2]. I defined a "behavior principle" [1, 7] according to which an experimenter performing quantum experiments cares about 
his descendants according to their measures of existence. This principle answers the (naive) criticism that a believer in the MWI would agree to play "Russian Quantum Roulette" [28]. "Measure of existence" is a philosophically problematic term, so "caring measure" is frequently used instead of "measure of existence" [29].

Albert [12] recently criticized this approach, providing deliberately ridiculous alternative measure (proportional to the measure of existence of the branch times fatness of the observer in this branch) and arguing that this caring measure is as good as the other one. Albert's criticism might apply if caring measure is considered as a standing alone proposal. In my approach the foundation of the caring measure is the post-measurement genuine probability of the descendants of the experimentalist. In a typical quantum experiment, splitting of worlds (creation of superposition of macroscopically different wavepackets of macroscopic systems) happens before the time the experimentalist (i.e. all of his versions) will become aware of the outcome. So, there will be a stage with genuine probability concept. At that moment all the descendants will be happy if actions according to the behavior principle have been performed.

I am encouraged by recent support coming from Tappenden [30], who approved attaching post-measurement uncertainty to (the illusion) of premeasurement probability naming it the Born-Vaidman Rule. Tappenden uses it for the analysis of confirmation of the MWI; see also Greaves and Myrvold [31]. I might agree with these arguments, but for me, the strongest confirmation of the MWI lays in the non-probabilistic consequences of quantum theory, such as spectrum of a hydrogen atom. Experiments confirming this type of predictions are so successful that only extreme deviation from the Born-Vaidman rule might question the MWI.

\section{Conclusions}

I have argued that there are two main issues related to probability in the MWI. First is how to talk about the probability of an outcome in a measurement when all outcomes are actualized, and the second is what is the status of the Born rule.

If we disregard the first problem, then the second is simple: The MWI tells us that All is the Wave Function. Locality tells us that the result of any experiment in one location can depend only on the property of the Wave 
Function in this location. The expectation value of the projection of the Wave Function on this location is the only property which cannot be changed by actions elsewhere, so causality tells us that the probability is the function of this projection. Then, from a symmetry argument and/or Gleason theorem the Born rule is derived.

The resolution of the first problem is the statement that there is no genuine probability concept in the MWI, and the "sleeping pill" argument explains well why we have an illusion of probability which is essentially indistinguishable from real probability. The combination of two arguments would solve the whole problem, but unfortunately, it requires additional, although very plausible, assumptions regarding what might influence the probability of an outcome. Note, however, that with such an assumption the Born rule can be derived in the framework of other interpretations of quantum theory as well.

I understand that Itamar and I viewed the problem of the Born rule in a similar way, i.e. that some assumptions are necessary in order to prove the Born rule using the Gleason theorem [32]. On the other hand, Itamar and Meir Hemmo were sceptical about resolution of the first issue. I believe that the reason why I do not see the difficulties they encountered is that I consider a direct connection between the Wave Function and our experience without insisting on giving values to various observables, the topic which was at the center of Itamar's research. His view on the MWI was according to the lines of the many minds interpretation [33] in which the situation is very different, since, a priori, pre-measurement uncertainty seems to be possible and it is a non-trivial fact that actually it is not. Since I never tried to introduce the pre-measurement uncertainty in the first place, I had no reason to be discouraged by this result.

This work has been supported in part by the Israel Science Foundation Grant No. 1125/10.

\section{References}

[1] L. Vaidman, About Schizophrenic Experiences of the Neutron or Why We Should Believe in Many-Worlds Interpretation of Quantum Theory, Preprint of University of South Carolina, http://www.tau.ac.il/ vaidman/lvhp/upm1.pdf (1990). 
[2] L. Vaidman, On Schizophrenic Experiences of the Neutron or Why We Should Believe in the Many-Worlds Interpretation of Quantum Theory, Int. Stud. Phil. Sci. 12, 245-261 (1998).

[3] G.C. Ghirardi, A. Rimini, and T. Weber, Unified Dynamics for Microscopic and Macroscopic Systems, Phys. Rev. D 34, 470-491 (1986).

[4] P. Pearle, Combining Stochastic Dynamical State-Vector Reduction with Spontaneous Localization, Phys. Rev. A 39, 2277-2289 (1989).

[5] D. Bohm, A Suggested Interpretation of the Quantum Theory in Terms of Hidden Variables, I and II, Phys. Rev. 85, 166-193 (1952).

[6] H. Everett III, 'Relative State Formulation of Quantum Mechanics, Rev. Mod. Phys. 29, 454-462 (1957).

[7] L. Vaidman, Many-Worlds Interpretation of Quantum Mechanics, Stan. Enc. Phil., E. N. Zalta (ed.) (2002), http://plato.stanford.edu/entries/qm-manyworlds/.

[8] Quantis, True Random Number Generator Exploiting Quantum Physics, http://www.idquantique.com/true-random-numbergenerator/products-overview.html/.

[9] Tel-Aviv World Splitter, http://qol.tau.ac.il/tws.html.

[10] T. Maudlin, Can the World Be Only Wavefunction?, in Many Worlds? Everett, Quantum Theory, and Reality, S. Saunders, J. Barrett, A. Kent, and D. Wallace (eds.) pp.121-143 (Oxford University Press 2010).

[11] D. Deutsch, Quantum Theory of Probability and Decisions, Proc. Royal Soc. Lon. A 455, 3129-3137 (1999).

[12] D. Albert, Probability in the Everett Picture, in Many Worlds? Everett, Quantum Theory, and Reality, S. Saunders, J. Barrett, A. Kent, and D. Wallace (eds.) pp.355-368 (Oxford University Press 2010).

[13] I. Pitowsky, Betting on the Outcomes of Measurements: a Bayesian Theory of Quantum Probability, Stud. Hist. Phil. Mod. Phys. 34, 395414 (2003). 
[14] Y. Aharonov and L. Vaidman, Nonlocal Aspects of a Quantum Wave, Phys. Rev. A 61, 052108 (2000).

[15] I. Pitowsky, De Finetti Extensions, Quantum Probability and Gleason's Theorem, in Probability is Physics ,C. Beisbart and S. Hartman (eds.) (Oxford University Press 2011).

[16] A. Elga, Self-locating Belief and the Sleeping Beauty Problem, Analysis 60, 143-147 (2000).

[17] L. Vaidman, and S. Saunders, On Sleeping Beauty Controversy, Oxford University Preprint, http://philsci-archive.pitt.edu/324/ (2001).

[18] L. Vaidman, Probability and the Many Worlds Interpretation of Quantum Theory, in Quantum Theory: Reconsidereation of Foundations, A. Khrennikov, (ed.) pp.407-422 (Vaxjo University Press, Sweden 2001).

[19] B. Groisman, The End of Sleeping Beauty's Nightmare Brit. J. Phil. Sci. 59, 409-416 (2008).

[20] P. Lewis, Quantum Sleeping Beauty, Analysis 67, 59-65 (2007).

[21] P. Lewis, Reply to Papineau and Dura-Vila, Analysis 69, 86-89 (2009).

[22] D.J. Bradley, Confirmation in a Branching World: The Everett Interpretation and Sleeping Beauty, Brit. J. Phil. Sci., doi:10.1093/bjps/axq013 (2011).

[23] D. Papineau and V. Dura-Vila, A Thirder and an Everettian: a Reply to Lewis's Quantum Sleeping Beauty, Analysis 69, 78-86 (2009).

[24] S. Saunders, Time, Quantum Mechanics, and Probability, Synthese 114, 373-404 (1998).

[25] D. Wallace, Epistemology Quantized: Circumstances in which We should Come to Believe in the Everett Interpretation, Brit. J. Phil. Sci. 57 655-689 (2006).

[26] D. Peterson, Qeauty and the books: a response to Lewis's Quantum Sleeping Beauty Problem, Synthese, doi:10.1007/s11229-010-9715-5 (2010). 
[27] J. Bertrand, Calcul des probabilités, (Paris: Gauthier-Villars et fils, 1889).

[28] P. Lewis, What is it like to be Schrödinger's cat? Analysis 60, 22-29 (2000).

[29] H. Greaves, Understanding Deutsch's Probability in a Deterministic Multiverse, Stud. Hist. Phil. Mod. Phys. 35, 423-456 (2004).

[30] P. Tappenden, Evidence and Uncertainty in Everett's Multiverse, Brit. J. Phil. Sci. 62, 99-123 (2011).

[31] H, Greaves and W. Myrvold, Everett and Evidence, in Many Worlds? Everett, Quantum Theory, and Reality, S. Saunders, J. Barrett, A. Kent, and D. Wallace (eds.) pp.264-307 (Oxford University Press 2010).

[32] M. Hemmo and I. Pitowsky, Quantum Probability and Many Worlds, Stud. Hist. Phil. Mod. Phys. 38, 333-350, (2007).

[33] M. Hemmo and I. Pitowsky, Probability and Nonlocality in Many Minds Interpretations of Quantum Mechanics, Brit. J. Phil. Sci. 54, 225-243 (2003). 